\title{
Lealtad Democrática de los Partidos de Oposición en América Latina (1978-2010): Una Explicación Institucionalista
}

\author{
Daniela Vairo ${ }^{1}$ \\ ${ }^{1}$ Professora no Instituto de Ciencia Política da Universidad de la República, Uruguai. Pesquisadora bolsista \\ (ANII, Uruguai). \\ E-mail: danielavairo@gmail.com, https:/ / orcid.org/0000-0003-4426-6460
}

\section{INTRODUCCIÓN}

T a pregunta que guía este artículo es: ¿bajo qué condiciones L las oposiciones partidarias latinoamericanas de la tercera ola son leales o desleales a la democracia? La lealtad democrática es una opción que se le presenta a todos los partidos políticos y especialmente a aquellos ubicados en la oposición (Vairo, 2017). Estos partidos no cuentan con las ventajas del espacio de poder principal en el presidencialismo, el Poder Ejecutivo, por lo que sus incentivos para cooperar son menores que entre quienes son parte del gobierno.

Este artículo procura contribuir a los estudios sobre partidos políticos de oposición y democracia en América Latina. Si la democracia alcanza el equilibrio cuando tanto ganadores como perdedores acatan los resultados electorales y sus consecuencias (Przeworski, 1991), se torna necesario un análisis más sistemático de los comportamientos de los partidos de oposición en las democracias latinoamericanas contemporáneas. La literatura establece que oposiciones poco comprometidas con el régimen democrático son problemáticas para la supervivencia de la democracia (Linz, 1987; O'Donnell et al., 1986). Trabajos más actuales como el de Gamboa (2017), muestran que los objetivos y tácticas de las oposiciones afectan no solo la probabilidad de quiebre, sino también las de erosión democrática.

Sin embargo, estos estudios no establecen bajo qué condiciones las oposiciones son leales o desleales al régimen. Es decir, la relevancia de los partidos opositores para la democracia no ha derivado en

DADOS - Revista de Ciências Sociais, Rio de Janeiro, vol.62(1):e20160173.

http://dx.doi.org/10.1590/001152582019171 
el desarrollo de un cuerpo teórico y un estudio sistemático sobre sus comportamientos en relación al propio régimen, y menos aún centrados en América Latina.

El argumento explicativo central de este artículo procura recuperar la tradición institucionalista. Ante la presencia de teorías que explican la democracia a través de dimensiones de la estructura económicodistributiva, esta investigación plantea volver al institucionalismo como eje explicativo central. La hipótesis central del trabajo es que la lealtad democrática de la oposición partidaria depende en gran medida del grado de inclusión/exclusión institucional a la que se enfrenta. En concreto, se plantea que diseños institucionales más incluyentes se ven acompañados de oposiciones partidarias leales, mientras que sistemas más excluyentes derivan en oposiciones desleales.

La exclusión institucional es entendida como configuración de reglas que, a través de la concentración/dispersión del poder político, permiten potencialmente a la oposición participar en la toma de decisiones públicas. Sistemas más excluyentes generarían oposiciones partidarias desleales a la democracia, mientras que sistemas incluyentes se relacionarían con oposiciones leales. La exclusión institucional es operacionalizada en tres dimensiones: i) el sistema electoral legislativo; ii) los poderes presidenciales y iii) la dispersión de poder institucional. Para cada una de ellas, se seleccionan indicadores que luego son utilizados para testear la teoría. A las hipótesis principales se agregan hipótesis alternativas, vinculadas a la estructura económica (riqueza, desigualdad, dependencia de recursos naturales) y al régimen político (tipo de régimen competitivo).

El resto del artículo se organiza en cuatro partes. En la próxima sección se conceptualiza y operacionaliza la variable dependiente: lealtad democrática de la oposición partidaria. A continuación, se desarrolla el argumento explicativo y sus dimensiones, para luego mostrar el test estadístico de las hipótesis a través de la estimación de modelos logit. Por último, se realizan algunas reflexiones finales.

\section{LA LEALTAD DEMOCRÁTICA DE LA OPOSICIÓN PARTIDARIA}

Este artículo procura estudiar la lealtad de la oposición partidaria en el marco de los regímenes competitivos presidencialistas de la tercera ola 
democrática latinoamericana. La teoría explicativa es elaborada con aplicación para las democracias latinoamericanas posdictadura. Como señalan Mainwaring y Pérez-Liñán (2009), las regiones tienen procesos políticos particulares (especificidades regionales) así como influencia intraregión (difusión regional). De esta forma, las características particulares regionales operan como "control" de la teoría ${ }^{1}$.

La lealtad democrática de los partidos de oposición ha sido un objeto de estudio bastante infrecuente en la Ciencia Política. Si bien algunos autores clásicos como Dahl (1966, 1973), Duverger (1957) o Sartori (1966) clasifican a las oposiciones según sus objetivos, estrategias y principios, es en el trabajo de Linz (1987) que se encuentra una referencia más directa y sistemática sobre la cuestión del compromiso democrático de las oposiciones y su efecto sobre el régimen político ${ }^{2}$.

De esta manera, con base en la obra de Linz (1987), aquí se define la lealtad democrática de la oposición partidaria como las actitudes respecto al régimen que denotan (o no) compromiso con las reglas, procedimientos y resultados inherentes a la democracia competitiva. En este sentido, una oposición partidaria leal es aquella que se adhiere al régimen político democrático, oponiéndose al gobierno de turno, pero no por ello al sistema. Este tipo de oposición, reconoce y acata los resultados electorales, respeta las decisiones del gobierno aun cuando estas no sean de su preferencia y espera a que los plazos constitucionales le den una nueva oportunidad para competir electoralmente (Vairo, 2017:281). Por el contrario, una oposición desleal corresponde a la negación total o parcial de las condiciones que caracterizan a una oposición leal. Es decir, son aquellas oposiciones que desconocen, atacan y/o desestabilizan al régimen competitivo (Vairo, 2017:281).

Este concepto general de lealtad de la oposición es tratado operacionalmente en este artículo como variable dependiente, tomando los datos del indicador de preferencia por la democracia de los partidos de oposición en regímenes competitivos ${ }^{3}$ entre 1978 y $2018^{4}$, disponible en Mainwaring y Pérez-Liñán, (2013) ${ }^{5}$.

El indicador de preferencia por la democracia mide la valoración intrínseca de la democracia y / o de la dictadura, más allá de las políticas públicas asociadas a un determinado momento histórico (Mainwaring y Pérez-Liñán, 2014). Siguiendo a los autores, los indicadores que se 
utilizan para categorizar a los partidos políticos como "sin preferencia por la democracia" son: i) la expresión de ambivalencia sobre la democracia; ii) de hostilidad sobre sus instituciones más relevantes (partidos, Parlamento, Poder Judicial, organismos electorales) más allá de decisiones concretas; iii) el cuestionamiento del valor de los procedimientos democráticos cuando les son desfavorables; y iv) la presentación del contrincante político como enemigo ilegítimo en lugar de contrincante electoral (Mainwaring y Pérez-Liñán, 2014:148). En cambio, es considerado que un actor prefiere la democracia cuando la valora intrínsecamente, está comprometido ideológicamente con ella, más allá de las políticas públicas asociadas a un determinado momento histórico (Mainwaring y Pérez-Liñán 2013, 2014).

En este artículo, el concepto y su operacionalización se definen en términos agregados, a nivel país-año. Es decir, se considera a la oposición como subsistema de partidos. Esto se debe a que el objetivo del trabajo es discernir si sistémicamente, la oposición partidaria varía en su lealtad según cambios en variables institucionales. Variables institucionales que, como se verá más adelante, se manifiestan a nivel de cada país en cada momento son confrontadas con los resultados a nivel de lealtad democrática en cada país-año. De esta manera, se mantiene el nivel de análisis "país-año", tanto para los factores explicativos como para la variable dependiente. Operacionalmente, esto implica que no se toman los partidos políticos de oposición en forma individual, sino que como el promedio simple del valor del indicador para los principales partidos de oposición ${ }^{6}$, en cada país-año (Vairo, 2017:282).

El Gráfico 1 muestra el valor promedio, para cada país y para todo el período, de la preferencia por la democracia de la oposición partidaria ${ }^{7}$. Es posible identificar tres grupos. En primer lugar, están aquellos países que desde la tercera ola han tenido mayoritariamente oposiciones partidarias leales: Argentina, Panamá, Costa Rica, Uruguay, República Dominicana y Brasil. Por ejemplo, Costa Rica durante el período de estudio tuvo un régimen competitivo, con un sistema de partidos estable, con alternancia de gobiernos entre el Partido Liberación Nacional y el Partido Unión Social Cristiana. Los partidos de oposición que fueron considerados para el cálculo de la preferencia por la democracia en cada año, además de estos dos últimos cuando no estuvieron en el gobierno, fueron el Partido Vanguardia Popular, el Partido Fuerza Democrática y, sobre el final del período, 
el Partido de Acción Ciudadana. En todos estos casos, los partidos fueron codificados como prodemocráticos por lo que las oposiciones partidarias en Costa Rica han sido casos claros de oposiciones leales (Vairo, 2017). Estos partidos se presentan a elecciones, respetan los procedimientos constitucionales y reconocen los resultados que lo colocan del lado de los perdedores (Castañeda-Angarita, 2011).

\section{Gráfico 1}

Preferencia por la democracia de la oposición partidaria (promedio por país 1978-2010)

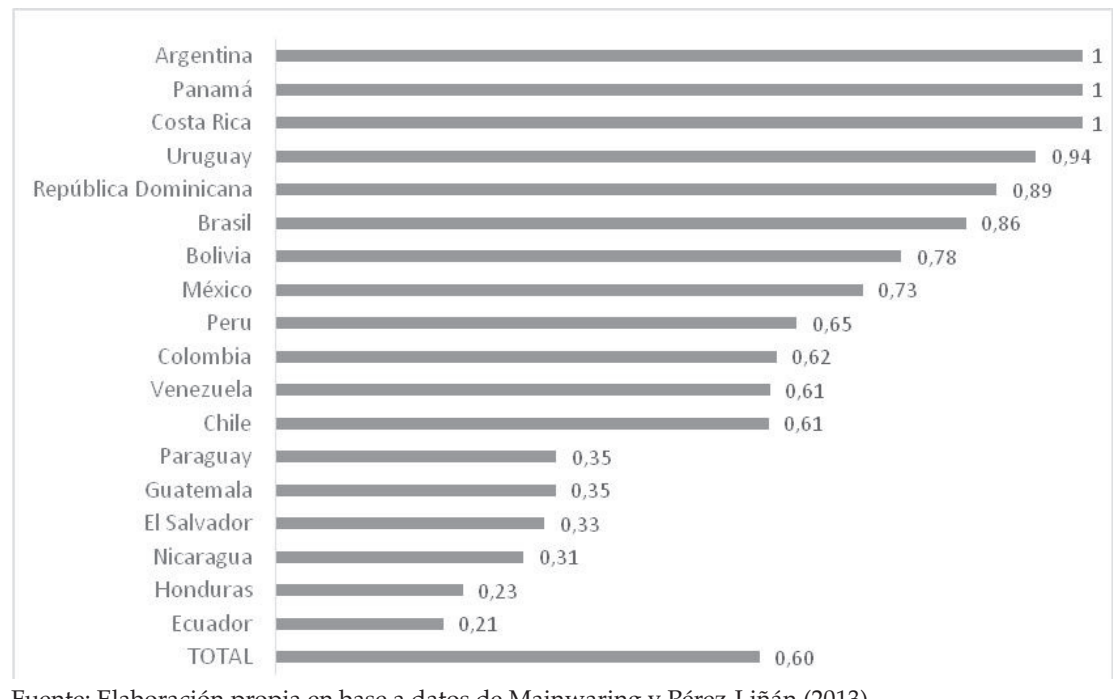

Fuente: Elaboración propia en base a datos de Mainwaring y Pérez-Liñán (2013).

En el otro extremo, el grupo de países conformado por Ecuador, Honduras, Nicaragua, El Salvador, Guatemala y Paraguay tienen oposiciones partidarias más bien desleales a la democracia en el promedio del período. Por ejemplo, en el caso de Ecuador los partidos de oposición han variado a lo largo de los años estudiados entre Izquierda Democrática, Partido Social Cristiano, Partido Roldosista Ecuatoriano y, sobre el final del período de análisis, el Partido Sociedad Patriótica. En general, estos partidos no se han mostrado comprometidos con la democracia, sino que más bien han sido pragmáticos, populistas y en varias ocasiones han intentado desestabilizar a los gobiernos electos. Son hostiles y valoran más los resultados políticos que las reglas democráticas (Vairo, 2017). Un ejemplo sería en 1996-1997 cuando la oposición intenta remover al presidente Abdalá Bucaram a través de un juicio político y como no consigue dos tercios de los votos necesarios del parlamento, utiliza 
argumentos pseudoconstitucionales y aduce que es mentalmente incapaz (García y Polga-Hecimovich, 2012).

El tercer grupo se compone de aquellos países que en promedio han tenido tanto oposiciones leales como desleales a lo largo del período. Forman parte de este grupo: Chile, Venezuela, Colombia, Perú, México y Bolivia. Por ejemplo, en el caso chileno el período de análisis es entre 1990 y 2010, ya que antes se encontraba bajo un régimen autoritario. Al inicio del período, había dos partidos principales de oposición: Unión Democrática Independiente y Renovación Nacional. La Unión Democrática Independiente tenía una fuerte identificación con el anterior régimen autoritario, una visión de la política antipartidos y tecnocrática, y proponía un régimen semicorporativo (Armesto, 2011). En la segunda parte del período, a partir de 2000 aunque en forma gradual, aumenta su compromiso democrático hasta convertirse en una oposición leal al régimen. En cambio, Renovación Nacional, es un partido de derecha que se ha desmarcado del gobierno dictatorial de Pinochet y ha mostrado apego público a las reglas democráticas.

\section{LA EXPLICACIÓN INSTITUCIONAL}

\section{Argumento teórico e hipótesis}

¿Bajo qué condiciones los partidos de oposición son leales o desleales a la democracia? La respuesta que propone este artículo es neoinstitucional (March y Olsen, 1984; Hall y Taylor, 1996) en el entendido de que las reglas y el contexto institucional moldean los incentivos de los actores e influyen en sus comportamientos.

¿En qué medida se reconoce institucionalmente a la oposición? Es decir, ¿qué reglas incluyen o excluyen la participación de los partidos de oposición en espacios de poder decisorio públicos? y ¿cuán concentrado o disperso está el poder político-institucional? En particular, lo que aquí se afirma es que el carácter inclusivo/excluyente de la configuración institucional, en términos de arreglos formales y distribución del poder, afectan la probabilidad de que la oposición partidaria esté comprometida o no con el régimen democrático.

Para que la oposición partidaria tenga incentivos para ser leal se requieren instituciones que generen expectativas a los perdedores 
de no ser tan perdedores, de ser ganadores parciales o al menos potenciales. Esta noción aquí es entendida como inclusividad de las instituciones. En la medida en que la oposición es reconocida institucionalmente (incluida), sus incentivos para la deslealtad democrática disminuyen, debido a que a pesar de estar del lado de los perdedores, sus opiniones, creencias e intereses están reflejados de algún modo en el ámbito político-público. Por el contrario, retomando a Linz: "la exclusión sistemática o la discriminación de los partidarios de la oposición en muchos campos de la vida pública (...) puede empujar a aquellos dispuestos a constituir una oposición leal a posturas semi o desleales" (Linz, 1987:68).

Los marcos institucionales que producen ganadores y perdedores absolutos no generan incentivos en los perdedores a apoyar un régimen que los desconoce (Colomer, 2001). Esto es particularmente importante en los regímenes presidencialistas como los latinoamericanos, donde el premio mayor es el cargo de presidente. Sin embargo, existe una enorme variación en la configuración institucional de los regímenes presidenciales que hace que en algunos sistemas el perdedor sea más perdedor que en otros, es decir, que la oposición partidaria esté más excluida que en otros ${ }^{9}$.

Existe una tensión entre representación y eficiencia/gobernabilidad y suele preferirse lo segundo para garantizar la estabilidad política. Se afirma que cuantos más actores con poder de veto existan, hay más posibilidades de parálisis gubernamental e inestabilidad. Sin embargo, como señala Coutinho: “(...) existem menores incentivos para se agir contra um regime do qual se participa por intermédio dos seus governos. A inclusão pressupoe partilha do poder, que por sua vez, pressupoe menos incentivos à conspiração" (2008:58). En el enfoque propuesto en este artículo, el aumento del número de actores en el sistema no genera inestabilidad, sino dispersión del poder e inclusión que llevan al compromiso, lo que aquí se define como lealtad.

De esta manera, la hipótesis central del artículo es que la lealtad democrática de la oposición partidaria depende en gran medida del grado de inclusión/exclusión institucional a la que se enfrenta. La inclusión/exclusión institucional es entendida como configuración de reglas y distribución del poder que permiten o no potencialmente a la oposición participar en centros de toma de decisiones públicas. Diseños institucionales más incluyentes se ven acompañados de 
oposiciones partidarias leales, mientras que sistemas más excluyentes derivan en oposiciones más desleales.

La inclusividad es operacionalizada a partir de tres dimensiones explicativas representadas en el Diagrama 1: i) el sistema electoral legislativo; ii) los poderes presidenciales; y iii) la dispersión de poder institucional.

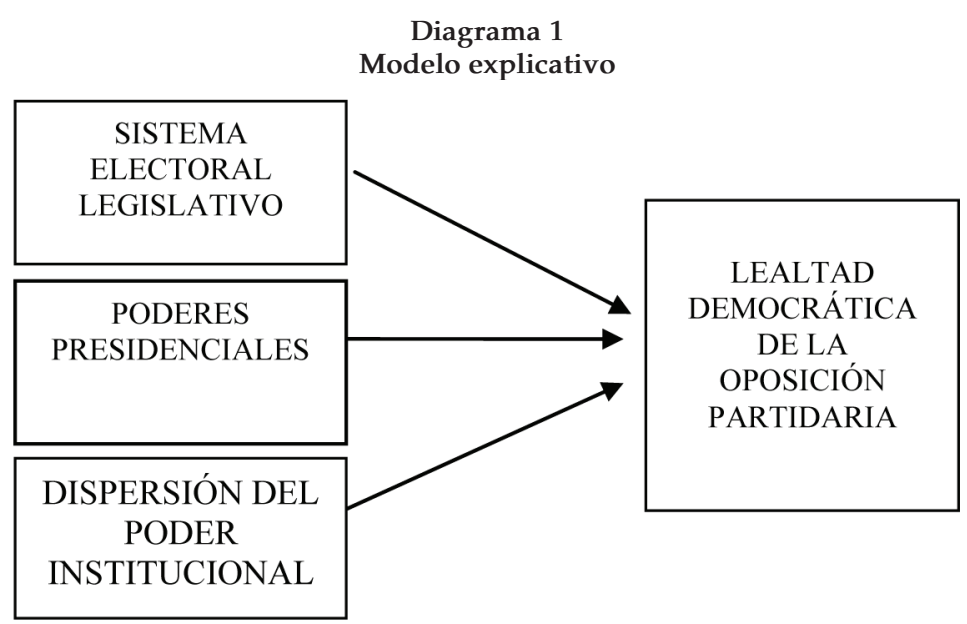

Fuente: Elaboración propia.

La hipótesis central se subdivide en tres hipótesis específicas:

H1) Cuanto más excluyente es el sistema electoral legislativo, menor probabilidad de lealtad democrática de la oposición partidaria.

H2) Cuanto mayores sean los poderes constitucionales del presidente, menor probabilidad de lealtad democrática de la oposición partidaria.

H3) Cuanto menor sea la dispersión del poder institucional, menor probabilidad de lealtad de la oposición partidaria.

Cada una de estas hipótesis puede ser reelaborada en forma inversa relacionando la inclusión con la deslealtad democrática de la oposición partidaria. Es decir, cuanto más excluyente es el sistema electoral legislativo, mayores incentivos para la deslealtad, y así sucesivamente. 
Primera dimensión explicativa: el sistema electoral legislativo

La primera dimensión explicativa es el sistema electoral legislativo. En los presidencialismos, el Parlamento es el principal centro de poder de la oposición partidaria ${ }^{10}$. Es así que las reglas de elección legislativa son fundamentales ya que definen qué proporción de los partidos de oposición se ve representada y la distribución del poder.

En esta investigación el sistema electoral será considerado en términos de reglas que potencialmente incluyen o excluyen a la oposición partidaria ${ }^{11}$. El mayoritarismo o proporcionalidad ${ }^{12}$ (representatividad a fin de cuentas) del sistema influye en los comportamientos de los partidos políticos de oposición a través de su carácter excluyente/incluyente.

Los sistemas mayoritarios por definición son excluyentes. Tienen virtudes asociadas a la efectividad y capacidad de gobierno, sin embargo, generan incentivos para la deslealtad de la oposición partidaria ya que esta ve reducida al mínimo la probabilidad de acceder al Parlamento y gozar de una bancada relevante, es decir, estos sistemas disminuyen la representación y legitimidad (ver por ejemplo Colomer, 2001). De esta forma, los partidos más grandes se ven favorecidos y el resto de agrupaciones políticas se ve perjudicado. En cambio, sistemas más proporcionales permiten la representación parlamentaria de distintas voces e intereses.

En este trabajo se toman en cuenta aquellas disposiciones que tienen un carácter incluyente/excluyente sobre la oposición partidaria para luego construir un índice que resume las diversas configuraciones de reglas. Se escogieron los siguientes indicadores del diseño electoral legislativo de las Cámaras Bajas ${ }^{13}$ : la magnitud promedio de circunscripción, la desproporcionalidad (índice de mínimos cuadrados), el tamaño de la asamblea (número de miembros de la cámara) y la existencia de un umbral electoral para el ingreso al Parlamento. Todos ellos dan cuenta de las restricciones institucionales para el acceso al Congreso. Se los toma en conjunto porque se entiende que operan como una configuración de reglas que actúan en conjunto.

Resumiendo esta primera dimensión explicativa (Tabla 1): una configuración institucional muy incluyente implica una magnitud 
de distrito promedio alta, baja desproporcionalidad, inexistencia de umbral de representación y un tamaño de asamblea alto. En el otro extremo, se encuentran los sistemas muy excluyentes, que son aquellos con magnitud de distrito promedio baja, alta desproporcionalidad, umbral de representación y tamaño de asamblea bajo.

Tabla 1

Inclusión/exclusión por indicador de sistema electoral legislativo

\begin{tabular}{lcc}
\hline Sistema electoral legislativo & Incluyente (0) & Excluyente (1) \\
\hline Desproporcionalidad & Baja & Alta \\
Magnitud distrito promedio & Alta & Baja \\
Umbral representación & No & $\mathrm{Si}$ \\
Tamaño asamblea & Alto & Bajo \\
\hline
\end{tabular}

Fuente: Elaboración propia.

\section{Los poderes presidenciales}

La segunda dimensión explicativa es la de los poderes formales del presidente. Dentro de los regímenes presidencialistas, los poderes institucionales del presidente son indicadores de concentración del poder político. Presidentes muy poderosos generan una dinámica política excluyente de la oposición partidaria, a través de la predominancia del Poder Ejecutivo sobre el Parlamento.

Shugart y Carey (1992) y Mainwaring y Shugart (2002) son pioneros en el estudio del presidencialismo latinoamericano. Ellos muestran que las variaciones institucionales dentro del presidencialismo son importantes y se centran en un aspecto: los poderes constitucionales de los presidentes ${ }^{14}$. Resumen estos poderes a partir de un índice muy popular en la disciplina (Shugart y Carey, 1992) que toma en cuenta los poderes legislativos y no legislativos de los presidentes (ver Tabla $2)^{15}$. Los poderes legislativos son el veto (parcial y total), el decreto ${ }^{16}$, la iniciativa legislativa exclusiva, los poderes presupuestarios y el poder para proponer referéndums (Shugart y Carey, 1992:148). 
Tabla 2

Índice de poderes presidenciales constitucionales

\begin{tabular}{ll}
\hline $\begin{array}{l}\text { Índice de poderes presidenciales } \\
\text { (Shugart y Carey, 1992) }\end{array}$ & \multicolumn{1}{c}{ Indicador } \\
\hline & $\begin{array}{l}\text { Veto (parcial/total y tipo de levantamiento u } \\
\text { "override") } \\
\text { Decreto }\end{array}$ \\
Poderes legislativos & Iniciativa exclusiva legislativa \\
& Poderes presupuesto \\
& Propuesta referéndum \\
\hline \multirow{2}{*}{ Poderes no legislativos } & Formación y despido gabinete \\
& Censura \\
\hline
\end{tabular}

Fuente: Elaboración propia en base a Shugart y Carey 1992:150.

Estos poderes, tomados en conjunto, afectan la capacidad de ejercicio de la oposición partidaria, de modo que cuanto más concentrado esté el poder en manos del presidente, se esperan mayores estímulos a su deslealtad democrática. Cuanto mayor sea el índice de poderes presidenciales más excluyente será el diseño institucional ${ }^{17}$.

\section{Dispersión de poder institucional}

Esta dimensión adapta el enfoque de Lijphart (1999) sobre diseños institucionales de las democracias mayoritarias y de consenso así como la teoría de los actores de veto de Tsebelis (2006). En el caso de Lijphart, este artículo procura captar la potencialidad de ciertas instituciones de las democracias de consenso, que se basan en la idea de que ganadores y perdedores tengan alguna cuota de poder. De la teoría de los jugadores de veto de Tsebelis se toma la idea de actores influyentes, con posibilidad de operar sobre la realidad política. La existencia de actores con amplios poderes y que éstos no estén alineados con el presidente dispersa el poder, señal de inclusividad. Estos actores influyentes son entendidos como ramas de poder, donde la oposición tiene posibilidad de ejercer algún dominio y contrarrestar el poder del presidente ${ }^{18}$.

En concreto, para trabajar esta dimensión explicativa en términos operacionales se optó por utilizar el índice de constreñimientos políticos denominado Polcon V (Henisz, 2000) que da cuenta de la dispersión del poder en diferentes actores institucionales relevantes 
tanto en términos de número (diseño), como su distribución real (ver Tabla 3$)^{19}$. Es decir, toma en cuenta los actores con posibilidad de limitar el cambio de políticas públicas y su alineación con el presidente (a través de la fraccionalización y el apoyo legislativo). En particular, los veto points que considera el índice son la existencia y poder real de una segunda cámara legislativa, entidades subfederales y Poder Judicial.

Tabla 3

Índice de constreñimientos políticos (Polcon V)

Índice de constreñimientos políticos Polcon V (Henisz, 2000)
Cámaras legislativas efectivas
Alineamiento partidario Ejecutivo-Legislativo
Fraccionalización legislativa
Federalismo efectivo
Poder Judicial independiente

Fuente: Elaboración propia en base a Henisz (2000).

Cuanto más alto sea el valor del índice de constreñimientos políticos, mayor dispersión del poder y más posibilidades de que la oposición partidaria tenga algún sitio de participación política, por lo que se espera que incentive su lealtad democrática. Al contrario, cuanto más bajo el valor del índice, mayor concentración del poder, menos posibilidades institucionales de participación de la oposición y mayores incentivos hacia la deslealtad.

\section{ANÁLISIS ESTADÍSTICO}

\section{Datos y métodos}

La base de datos es de 479 casos país-año correspondiente a los años con democracia competitiva de 18 países latinoamericanos entre 1978 y 2010. El modelo explicativo propuesto en la sección anterior se basa en la configuración institucional de los países en cada año y sus efectos sobre la probabilidad de que la oposición partidaria sea leal o desleal a la democracia. Para ello, se transforma el indicador de preferencia por la democracia en una variable dependiente binaria: son clasificadas como leales aquellas oposiciones que asumen un valor mayor a 0,5 
en el indicador de preferencia por la democracia y como desleales aquellas oposiciones que presentan un valor de 0,5 o menos.

Dado que la variable dependiente es discreta y dicotómica (oposición leal o desleal), para el análisis estadístico se utilizan modelos de regresión logística (método de máxima verosimilitud) de tipo logit. En este caso los modelos logit estimarán la probabilidad de que una oposición partidaria en un determinado país y año sea leal o desleal de acuerdo a un conjunto de variables independientes.

Además de las tres hipótesis principales que derivan del modelo explicativo (que involucran al sistema electoral legislativo, los poderes presidenciales y la dispersión del poder), se incorporan algunas hipótesis rivales que dan cuenta de posibles explicaciones alternativas. Es el caso del enfoque estructural distributivo proveniente de la economía política ${ }^{20}$. Las configuraciones institucionales y de distribución del poder en las que se centra la teoría de este trabajo operan en contextos económico-estructurales más o menos complejos que podrían agudizar o amortiguar los efectos de la inclusión/ exclusión institucional.

Aquí se seleccionan algunas variables clásicas como la riqueza ${ }^{21}$, la desigualdad ${ }^{22}$, y la estructura productiva dependiente del petróleo y minerales $^{23}$. Bajo estos enfoques se afirmaría que países más pobres (medido a través del PBI per cápita), con altos niveles de desigualdad (medido a través del Índice de Gini) y/o con gran dependencia de los recursos naturales (medido a través de la proporción de exportaciones sobre el ingreso bruto que provienen de este rubro) generarían mayores incentivos a la deslealtad de la oposición.

Se incluyen además dos variables de control. La primera se vincula al régimen político: el status del régimen en el año de estudio. Se entiende que el tipo de régimen en el que viven las oposiciones podría influir en el comportamiento de la oposición, de manera que regímenes competitivos plenos favorecerían la lealtad y regímenes competitivos con limitaciones ("semidemocráticos") se relacionarían con la deslealtad ${ }^{24}$. El argumento sería que el costo de la deslealtad es más alto en sistemas donde los derechos y libertades se respetan plenamente. Por último, se incorporará una variable de control que da cuenta de las expectativas de alternancia de los actores en el corto plazo. Es plausible considerar que las expectativas de alternancia 
puedan afectar los incentivos de la oposición partidaria de modo que, cuando las elecciones presidenciales se acercan, tienen estímulos para comprometerse con el régimen y sus reglas de juego. Sin embargo, al inicio del gobierno, la oposición partidaria puede verse desmotivada a actuar en forma comprometida y moderada.

La Tabla 4 muestra, para cada variable, el número de observaciones, valor mínimo y máximo, así como la mediana (variables numéricas) y moda (variables categóricas). Asimismo, se expresa el signo esperado de los coeficientes según las hipótesis ya esbozadas.

Tabla 4

Estadísticos resumen y signos esperados

\begin{tabular}{|c|c|c|c|c|c|c|c|}
\hline Variables & $\begin{array}{l}\text { Nombre } \\
\text { variable }\end{array}$ & $\begin{array}{c}\text { Signo } \\
\text { Esperado } \\
\text { sobre } \mathrm{P}(\mathrm{y}=1)\end{array}$ & $\mathrm{N}$ & Mediana & Moda & Min & Max \\
\hline \multicolumn{8}{|l|}{ Variable Dependiente } \\
\hline Lealtad oposición & lealtad & & 479 & - & 1 & 0 & 1 \\
\hline \multicolumn{8}{|l|}{$\begin{array}{l}\text { Variables } \\
\text { independientes }\end{array}$} \\
\hline Exclusión legislativa & exclusionleg & - & 479 & 2 & - & 0 & 4 \\
\hline Poderes presidenciales & poderespres & - & 479 & 16 & - & 6 & 25 \\
\hline $\begin{array}{l}\text { Constreñimientos } \\
\text { políticos }\end{array}$ & constrepol & + & 479 & 0.5 & - & 0,1 & 0,9 \\
\hline \multicolumn{8}{|l|}{ Variables de control } \\
\hline $\mathrm{PBI} /$ cápita & PBIcap_1 & + & 479 & 7.8 & - & 6,4 & 9,2 \\
\hline Gini & gini & - & 479 & 52 & - & 40,5 & 63,7 \\
\hline Petróleo y minerales & petroleoymin & - & 479 & - & 0 & 0 & 1 \\
\hline Semidemocracia & semidemoc & - & 479 & - & 0 & 0 & 1 \\
\hline $\begin{array}{l}\text { Años para fin de } \\
\text { gobierno }\end{array}$ & añosfingob & - & 479 & 2 & - & 0 & 5 \\
\hline
\end{tabular}

Fuente: Elaboración propia.

\section{Estimación de modelos y resultados}

Se estimaron cinco modelos logit (Tabla 5) con errores típicos robustos ${ }^{25}$. Se reportan los coeficientes para cada variable independiente en relación a la probabilidad de que una oposición partidaria sea leal $(y=1)$. 
Como la preferencia por la democracia es una variable que es estable en el tiempo en varios de los países estudiados, sería presumible que acarree características estructurales que podrían estar correlacionadas también con los regresores. Para testear esta presunción se estimó un modelo de regresión lineal con los mismos regresores que en el modelo completo logit pero introduciendo dummies por país (disponible en el Anexo Metodológico). Se constató que los resultados son similares a los del modelo original logit, lo que muestra que los hallazgos encontrados son efecto de las variables de interés y no un efecto de las características estructurales de los países ${ }^{26}$.

En el primer modelo, la lealtad democrática es modelada en función de las teorías rivales (económicas, del régimen y de expectativas). Los modelos 2, 3 y 4, incorporan, además, las variables independientes principales de la teoría en forma separada (exclusión legislativa, poderes presidenciales y constreñimientos políticos). El quinto modelo incluye todas las variables.

Tabla 5

Estimación de modelos

\begin{tabular}{lllllll}
\hline Dimensiones & Variable & Modelo1 & Modelo2 & Modelo3 & Modelo4 & Modelo5 \\
\hline $\begin{array}{l}\text { Exclusión y } \\
\text { concentración }\end{array}$ & exclusionleg & --- & $-0,265^{* *}$ & --- & --- & $-0,278^{* *}$ \\
del poder & $\begin{array}{l}\text { poderespres } \\
\text { constrepol }\end{array}$ & --- & --- & $-0,065$ & --- & $-0,084$ \\
\hline \multirow{2}{*}{$\begin{array}{l}\text { Económico- } \\
\text { estructural }\end{array}$} & PBIcap_1 & $2,072^{* * *}$ & --- & -- & $1,613^{* *}$ & $1,461^{*}$ \\
\hline Régimen & petroleoymin & $-0,132$ & $-0,149$ & $2,144^{* * *}$ & $2,000^{* * *}$ & $2,164^{* * *}$ \\
político & semidemoc & $-0,589^{*}$ & $-0,599^{*}$ & $-0,641^{*}$ & $-0,501$ & $-0,609^{*}$ \\
\hline Expectativas & añosfingob & $-0,158$ & $-0,139$ & $-0,165$ & $-0,147$ & $-0,136$ \\
\hline $\begin{array}{l}\text { Ajuste } \\
\text { modelos }\end{array}$ & r2_p & 0,275 & 0,286 & 0,279 & 0,286 & 0,3 \\
& Bic & 504,9 & 504,2 & 508,7 & 503,8 & 507,3 \\
\hline
\end{tabular}

$\mathrm{N}=479$

${ }^{*} \mathrm{p}<0.05 ;{ }^{* *} \mathrm{p}<0.01 ;{ }^{* *} \mathrm{p}<0.001$

Dentro de las teorías rivales (modelo 1), tanto el PBI como la semidemocracia muestran una asociación significativa con la lealtad democrática. Países más ricos tienden a tener oposiciones comprometidas con la democracia. Por su parte, los regímenes 
competitivos con alguna restricción (no plenos) desincentivan la lealtad, es decir, vivir bajo un régimen democrático pleno parece "proteger" de oposiciones desleales. Ni la desigualdad, ni la dependencia del petróleo y los minerales, ni el tiempo faltante para las siguientes elecciones son predictores significativos de la lealtad.

Si se observan los resultados de las variables independientes de exclusión institucional a partir del poder explicativo general, se puede afirmar que tanto la exclusión legislativa (H1) como la distribución del poder medida a través del índice de constreñimientos políticos (H3) tienen un efecto significativo sobre los extremos de las categorías de la variable. Esto puede constatarse tanto en los modelos en los que se testea su efecto individual (modelos 2, 3 y 4), como en el modelo completo (modelo 5). Los cambios en los niveles de exclusión legislativa o en la distribución del poder (a través del índice de constreñimientos políticos) tienen un efecto significativo en la probabilidad de que la oposición partidaria sea leal o desleal. En cambio, se descarta un efecto significativo de los poderes presidenciales (H2). Esto implica que la lealtad de la oposición partidaria no parece depender de la cantidad de poder institucional formal de los presidentes.

En resumen, los hallazgos indican que para entender por qué la oposición partidaria de un país en un año dado es desleal al régimen democrático es importante saber cuán excluidos están institucionalmente y cuán concentrado está el poder político en general (más en término de veto players que de poderes presidenciales constitucionales formales).

\section{Análisis de acuerdo a probabilidades predichas}

Las probabilidades predichas permiten un análisis más claro e intuitivo del efecto de las variables independientes sobre la variable dependiente. Fijando los valores de algunas o todas las variables independientes de interés (y dejando el resto en su valor medio), se pueden observar los cambios en la probabilidad de que la oposición sea leal. En este apartado, se trabajará con las dos variables independientes de la teoría que arrojan coeficientes significativos en el modelo completo (modelo 5): el índice de exclusión legislativa y el índice de constreñimientos políticos. 
El Gráfico 2 muestra que la probabilidad de encontrar una oposición partidaria leal disminuye a medida que va aumentando el índice de exclusión legislativa; pasa de 0,73 en los sistemas más inclusivos a 0,51 en los más excluyentes. En los sistemas con máxima exclusión legislativa (y el resto de las variables en su media) la probabilidad de encontrar una oposición leal o una desleal es casi la misma, mientras que en los sistemas más incluyentes es mucho más probable encontrar una oposición leal que una desleal (7 en 10).

Gráfico 2

Probabilidad de oposición leal según índice de exclusión legislativa

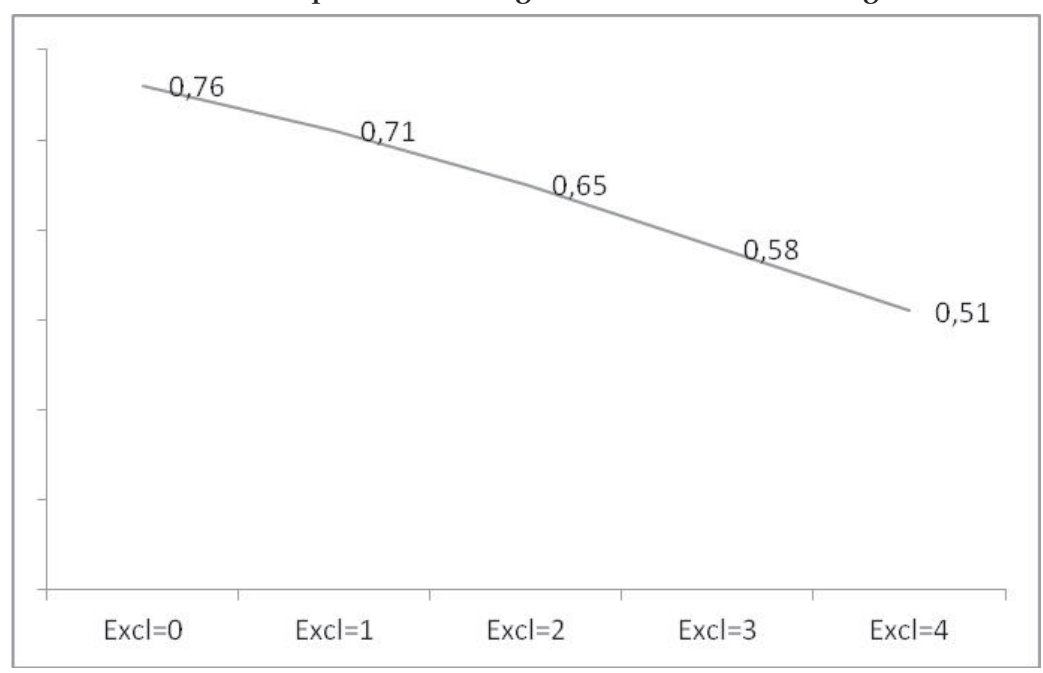

Fuente: Elaboración propia.

Algo similar sucede con el índice de constreñimientos políticos (Gráfico 3). Cuando este asume su valor más bajo, es decir, en los casos con máxima concentración de poder, la probabilidad de ser leal es tan solo de 0,52. A medida que aumenta la dispersión del poder, va aumentando la probabilidad de ser leal hasta llegar a 0,78. Es decir, la probabilidad de ocurrencia varía 0,26 entre el escenario de máxima concentración y el de máxima dispersión. 
Gráfico 3

Probabilidad de oposición leal según índice de constreñimientos políticos

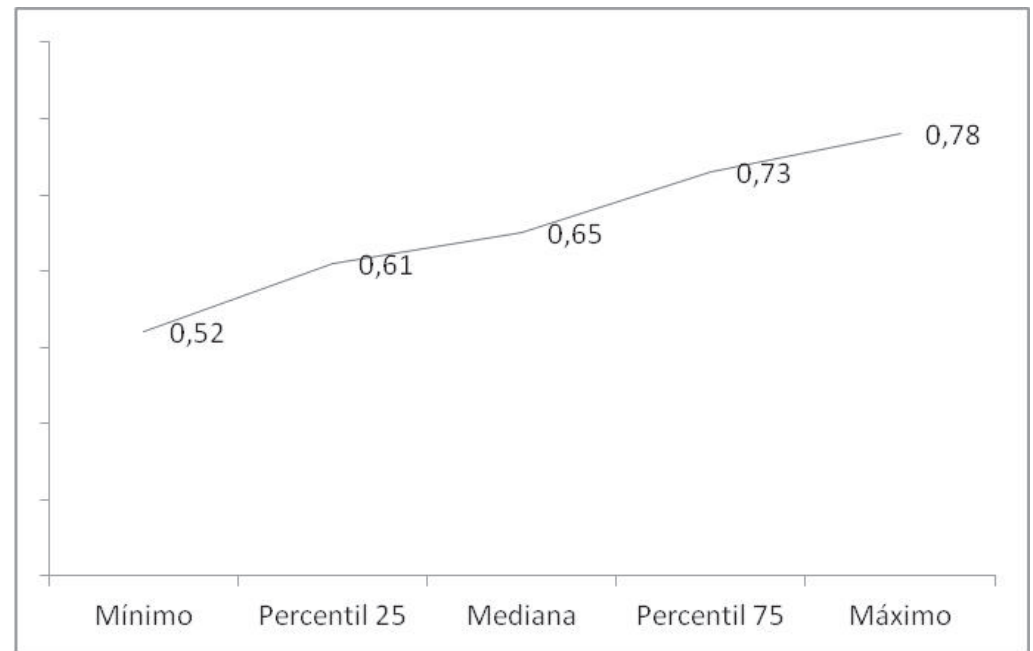

Fuente: Elaboración propia.

Simulación de escenarios incluyentes/excluyentes

A través de las simulaciones se fijan los valores de las variables de interés y se analiza la probabilidad predicha de ser leal o desleal para cada perfil. Se plantean cinco escenarios (Tabla 6) en los que se modifican las variables independientes de interés y se mantienen fijas en su valor promedio las de control. El primer y último escenario muestran, respectivamente, los efectos de las configuraciones de máxima inclusión y de máxima exclusión institucional. Esto surge de fijar simultáneamente en sus valores extremos los valores de exclusión legislativa, poderes presidenciales y constreñimientos políticos. Los escenarios 2, 3 y 4 fijan las variables de interés en los percentiles 25,50 y 75 , siguiendo el signo esperado de la asociación.

Tabla 6

Valores de las variables independientes fijados para cada escenario

\begin{tabular}{lccccc}
\hline Variable & $\begin{array}{c}\text { 1. Escenario } \\
\text { incluyente } \\
\text { máximo }\end{array}$ & $\begin{array}{c}\text { 2. Escenario } \\
\text { incluyente }\end{array}$ & $\begin{array}{c}\text { 3. Escenario } \\
\text { medio }\end{array}$ & $\begin{array}{c}\text { 4. Escenario } \\
\text { excluyente }\end{array}$ & $\begin{array}{c}\text { 5. Escenario } \\
\text { excluyente } \\
\text { máximo }\end{array}$ \\
\hline Exclusionleg & Min & Perc25 & Perc50 & Perc75 & Max \\
Constrepol & Max & Perc75 & Perc50 & Perc25 & Min \\
Poderespres & Min & Perc25 & Perc50 & Perc75 & Max \\
PBIcap_1 & Media & Media & Media & Media & Media
\end{tabular}




\begin{tabular}{llllll} 
Gini & Media & Media & Media & Media & Media \\
Petroleoymin & Media & Media & Media & Media & Media \\
Semidemoc & Media & Media & Media & Media & Media \\
Añosfingob & Media & Media & Media & Media & Media \\
\hline Fuente: Elaboración propia.
\end{tabular}

El Gráfico 4 muestra la probabilidad de encontrar una oposición partidaria leal o desleal a la democracia según los escenarios explicitados en la Tabla 6. El efecto de las variables independientes de la teoría, operando en conjunto, es muy fuerte. La probabilidad predicha de que exista una oposición partidaria leal es muy baja $(0,2)$ en escenarios de máxima exclusión institucional y es muy alta $(0,9)$ con escenarios de máxima inclusión. Por tanto, es muy difícil encontrar oposiciones leales en regímenes con valores extremos de exclusión legislativa, concentración del poder y poderes presidenciales.

Es interesante destacar que el aumento de la exclusión desde sus valores mínimos hasta sus valores medios (escenarios 1 a 3 ) implica una menor probabilidad de oposiciones leales y, por consiguiente, una mayor probabilidad de oposiciones desleales. Sin embargo, esto sucede en menor medida que los cambios que se generan en el pasaje de los escenarios 3 a 5 , es decir, al pasar de los valores medios de exclusión a los altos y extremos (escenarios de 3 a 5). En este caso, la probabilidad de enfrentarse a oposiciones desleales se duplica: de 0,4 en el escenario 3 (promedio) a 0,8 en el escenario 5 (máxima exclusión).

\section{Gráfico 4}

Probabilidad de lealtad o deslealtad según escenarios simulados

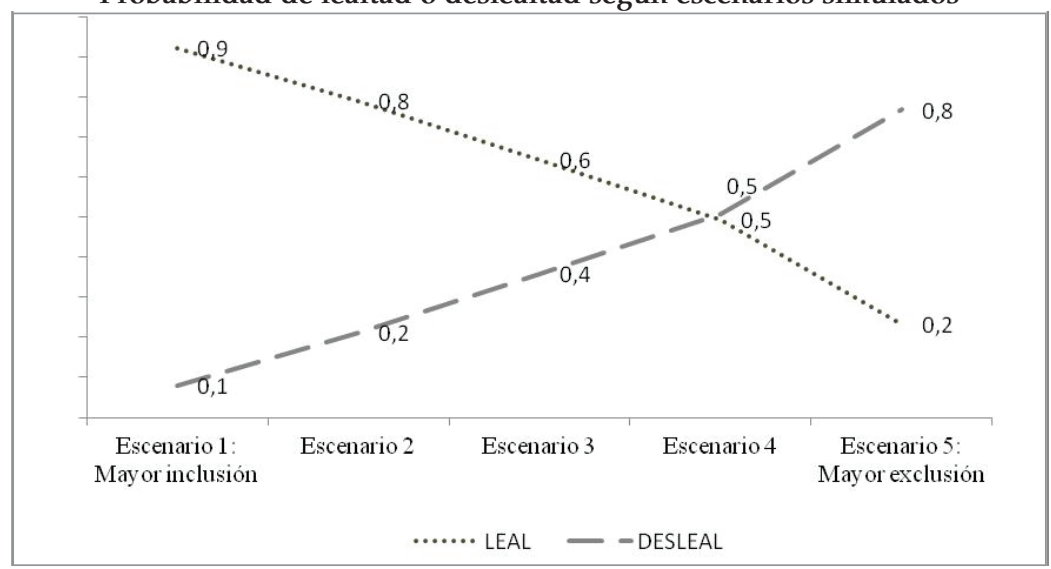

Fuente: Elaboración propia. 


\section{Discusión general de los hallazgos}

Elmodeloexplicativo predicecorrectamentelos resultados en términos de lealtad democrática de la oposición partidaria. Los hallazgos observados en las simulaciones con escenarios son contundentes: a mayor exclusión, mayor deslealtad y, por tanto, menor lealtad. En general, la exclusión institucional de la oposición partidaria, medida a través del índice de exclusión electoral legislativa (H1) y del índice de constreñimientos políticos (H3), tiene los efectos esperados sobre el fenómeno en estudio. No ocurre lo mismo con los poderes presidenciales $(\mathrm{H} 2)$ : no afectan significativamente la probabilidad de lealtad o de deslealtad. En este sentido, en futuras investigaciones sería interesante incorporar nuevos enfoques sobre la medición de los poderes del presidente (Doyle y Elgie, 2016).

Si se toma el efecto de cada variable de las hipótesis principales, el índice de exclusión legislativa (H1) y el índice de constreñimientos políticos (H3) tienen un impacto significativo y fuerte sobre la lealtad. Esto significa que en sistemas con exclusión institucional legislativa y poder concentrado en pocos actores relevantes (alineados al presidente), la oposición partidaria tiende a perder compromiso con la democracia (es decir, se inclina a ser desleal). En cambio, en los escenarios de mayor cantidad de actores institucionales relevantes y poder disperso la oposición tiende a ser leal al régimen.

En segundo lugar, es importante reconocer el poder explicativo de algunas de las variables de control, lo cual no contradice la teoría aquí propuesta, sino que la fortalece. Aun controlado por variables que suelen ser significativas para explicar fenómenos vinculados (como la estabilidad democrática), el poder explicativo de la exclusión institucional se mantiene. De igual modo opera la variable de control del régimen: sistemas que violan parcialmente alguno de los principios del régimen democrático pleno (es decir, aquellos que son categorizados como semidemocráticos), agudizan la problemática de la deslealtad en contextos excluyentes como los señalados por la teoría.

\section{CONSIDERACIONES FINALES}

La pregunta central que orientó este artículo fue: ¿bajo qué condiciones las oposiciones partidarias latinoamericanas de la tercera 
ola son leales o desleales a la democracia? La respuesta planteada intenta recuperar la tradición institucionalista para la explicación de fenómenos relacionados con los partidos políticos y la democracia.

El principal aporte que se procuró realizar con esta investigación es el de contribuir a la construcción de una teoría sobre la lealtad democrática de la oposición partidaria. Es una cuestión relevante para la disciplina y para los regímenes latinoamericanos, sin embargo no ha sido suficientemente conceptualizada o elaborada una teoría sobre su variación. Para ello se propuso una conceptualización operacional de la lealtad de los partidos de oposición a través de un indicador de preferencia por la democracia.

Tal como se podría esperar en regímenes competitivos, los casos leales son mayoritarios (60\%). Se expresan en partidos que pierden las elecciones, pero que prefieren la democracia ante cualquier otro tipo de régimen, por lo que respetan sus procedimientos y reglas básicas. Esto no significa que necesariamente cooperan con el gobierno, sino que respetan las instituciones, el resultado electoral y los canales y tiempos democráticos para el procesamiento de las decisiones políticas. Por su parte, las oposiciones desleales son aquellas que muestran un bajo o nulo compromiso normativo con la democracia y representan una minoría de los casos (40\%).

Asimismo, se formuló un modelo teórico simple sobre la variación de la lealtad que toma la exclusión institucional como principal explicación. En este sentido, ante la presencia cada vez más fuerte de teorías que explican las variables vinculadas a la democracia a través de dimensiones de la estructura económico-distributiva, este artículo plantea volver al institucionalismo como principal enfoque explicativo. La hipótesis central del trabajo es que la lealtad democrática de la oposición partidaria depende en gran medida del grado de inclusión/ exclusión institucional a la que se enfrenta. En concreto, se plantea que diseños institucionales más incluyentes se ven acompañados de oposiciones partidarias leales, mientras que sistemas más excluyentes derivan en oposiciones desleales.

Por último, se testean estadísticamente las hipótesis derivadas de la teoría. Se estiman modelos logit, controlando con las variables alternativas y utilizando la lealtad democrática de la oposición partidaria como variable dependiente con dos valores (leal y desleal). 
Los hallazgos generales indican que dos de las tres principales hipótesis (aquellas vinculadas al sistema electoral legislativo y a la dispersión del poder) tienen un poder explicativo sustantivo sobre la lealtad. En cambio, la variación en los poderes presidenciales no tiene un efecto significativo sobre la posibilidad de encontrar oposiciones leales o desleales a la democracia.

Algunas variables explicativas de control mostraron también tener un poder explicativo considerable. Particularmente, muestran coeficientes significativos el PBI per cápita y el tipo de régimen político, mientras que se descarta el efecto de la desigualdad y de la dependencia del petróleo y minerales. Países con democracia plena y mayor PBI se asocian menos con oposiciones partidarias desleales que aquellos países pobres y semidemocráticos.

¿Qué implicancias teóricas tienen estos hallazgos? El estudio a través de configuraciones institucionales se muestra provechoso para explicar la lealtad democrática de la oposición partidaria, aun controlado por variables estructurales y del régimen. Las instituciones, expresadas en términos de exclusión o inclusión, que conllevan concentración o dispersión del poder, tienen un importante poder explicativo. Los hallazgos observados en las simulaciones con escenarios son contundentes: a mayor exclusión, mayor deslealtad y, por tanto, menor lealtad. Las implicancias prácticas de la teoría refieren al diseño de reglas y políticas incluyentes que puedan llevar a oposiciones más democráticas lo que llevaría a una mayor calidad y estabilidad del régimen.

Si bien este artículo tiene alcance empírico en América Latina, se torna fundamental estudiar las implicancias de estos hallazgos para otras regiones. ¿Es la exclusión institucional un factor explicativo relevante para la existencia de oposiciones desleales en regiones más desarrolladas? ¿Es un patrón que solo se encuentra en regímenes presidenciales latinoamericanos? La esencia del argumento, que es que excluir institucionalmente a los opositores genera deslealtad, es aplicable teóricamente a cualquier tipo de régimen de gobierno o región. Para testearlo empíricamente en regímenes de gobierno parlamentarios o semi-presidencialistas, se tendrían que realizar algunos ajustes relativos al tipo de reglas característico de cada tipo régimen. 


\section{Daniela Vairo}

Por último, otro desafío para esta línea de investigación, fuertemente vinculado al anterior, es profundizar en el estudio de pocos casos, que puedan permitir comprender las dinámicas detrás de la (des)lealtad, tomando en cuenta el contexto histórico y otras variables propias de cada país.

(Recebido para publicação em 15 de agosto de 2016)

(Reapresentado em 4 de fevereiro de 2019)

(Aprovado em 14 de fevereiro de 2019)

\section{NOTAS}

1. Para ver una defensa de los estudios regionales en política comparada consultar Mainwaring y Pérez-Liñán (2009).

2. Vairo (2017) da cuenta de los aportes teóricos de Linz (1987[1978]) en torno a las oposiciones leales y desleales y propone una tipología de lealtad partidaria de la oposición que es analizada empíricamente para América Latina a partir de la tercera ola.

3. Sólo se toman en cuenta los años en que cada país tiene una democracia competitiva. El resto de los años no son considerados. Por ejemplo, esto implica que Uruguay tiene observaciones a partir de 1985, ya que antes su régimen era autoritario desde 1973.

4. El período de estudio seleccionado (1978-2010) obedece a la disponibilidad de datos de la variable dependiente en Mainwaring y Pérez-Liñán (2013).

5. En la base de datos original de Mainwaring y Pérez-Liñán (2013) estaba codificada la "preferencia por la democracia" de actores relevantes de diferente índole (gobierno, oposición partidaria y no partidaria y otros actores sociales y políticos). Para este trabajo, se seleccionaron únicamente los datos referidos a los partidos políticos de oposición. El proceso detallado de codificación y construcción de la variable dependiente a partir de Mainwaring y Pérez-Liñán (2013) puede consultarse en el Apéndice Metodológico.

6. Mainwaring y Pérez-Liñán (2013) seleccionan los principales actores políticos de acuerdo a fuentes historiográficas de cada país. Para este artículo, se extrajo la codificación del indicador de preferencia por la democracia únicamente referida a los partidos de oposición, dejando de lado otros actores relevantes en cada período (gobierno, Iglesia, Ejército, etc). Ver Apéndice Metodológico.

7. El indicador varía entre 0 y 1 . Cada actor es codificado por Mainwaring y Pérez-Liñán (2013) según tres valores: 0 si no es demócrata, 0,5 si es ambiguo o con posiciones internas divididas, y 1 si es demócrata. En el Gráfico se presenta el promedio por país para todo el período.

8. Esto no implica que en estos países no haya partidos de oposición u otros actores políticos poco comprometidos con la democracia, sino que se afirma que el subsistema de partidos de oposición más relevante, es mayoritariamente leal. 
9. Y todo esto se traduce en dinámicas diferentes del presidencialismo latinoamericano, como por ejemplo trabaja Lanzaro al referirse a los tipos de presidencialismo pluralista vs. presidencialismo mayoritario $(2001,2012)$. En la línea del argumento explicativo de este trabajo, la clasificación se vincula al pluralismo político y al grado de concentración o dispersión del poder (Lanzaro, 2012:23).

10. En los países federales el cargo de Gobernador también es un cargo muy relevante para políticos de todos los partidos.

11. El argumento sobre cuáles serían las reglas electorales de elección del Parlamento inclusivas o excluyentes es extraído de Buquet (2007), quien lo utiliza para explicar los incentivos de los actores para impulsar reformas electorales.

12. Para profundizar en las visiones mayoritarias y proporcionales, se sugiere consultar a Powell (2000).

13. Con fines comparativos se optó por trabajar con el sistema electoral legislativo de las Cámaras Bajas (o eventualmente única Cámara, si así correspondiera). La relevancia de la existencia de una segunda Cámara es captada por la tercera dimensión explicativa a través del Índice de Constreñimientos Políticos (Polcon V).

14. Aquí no se tomarán en cuenta los poderes partidarios de los presidentes: apoyo parlamentario y disciplina partidaria. El primero es captado por la tercera dimensión explicativa que se verá en la próxima sección, dentro del índice de dispersión institucional seleccionado. El segundo, no parece relevante para explicar el comportamiento (des)leal de la oposición partidaria.

15. Algunos autores ofrecen miradas alternativas, como Metcalf (2000); Aninat (2006); García Montero (2008) y Morgenstern, Polga-Hecimovich y Shair-Rosenfield (2013).

16. Para más detalle sobre el poder de decreto, véase Carey y Shugart (1998).

17. El índice de Shugart y Carey (1992) es la sumatoria de los puntajes de 10 indicadores de estos poderes presidenciales. Agradezco especialmente a Aníbal Pérez-Liñán y a María José Romero por brindarme los datos actualizados del índice.

18. En este sentido, Pérez-Liñán et al. (2019) muestran que la concentración del poder en manos del Ejecutivo, entendido como su control sobre el Poder Legislativo y sobre el Poder Judicial, es un factor explicativo fundamental para la erosión y caída de las democracias latinoamericanas de la tercera ola.

19. El objetivo de quienes elaboraron el índice es, a la Tsebelis, estudiar los efectos de los ambientes políticos que limitan la probabilidad del cambio de políticas. Particularmente, "...the political constraint index does directly measure the feasibility of a change in policy given the structure of a nation's political institutions (the number of veto points) and the preferences of the actors that inhabit them (the partisan alignment of various veto points and the heterogeneity or homogeneity of the preferences within each branch)" (Henisz, 2002).

20. Traversa (2016) realiza una extensa revisión y discusión de la literatura estructuralista que procura explicar variables relacionadas al régimen político (democratización, estabilidad, supervivencia de la democracia). Además, puede consultarse a Munck (2010). 


\section{Daniela Vairo}

21. La teoría de la modernización que señala que existe una fuerte asociación entre desarrollo económico y democracia. Ver por ejemplo Lipset, 1959; Przeworski et al., 1997; Przeworski, 2005.

22. Para profundizar en las teorías distributivas ver por ejemplo Boix, 2003; Acemoglu y Robinson, 2006.

23. Esta literatura señala que los países con altos ingresos petroleros se vinculan negativamente con la democracia (Karl, 1997; Ross, 2001). Como señala Ross, la renta petrolera, es decir, la riqueza basada en el petróleo hace a los países menos democráticos (2001:330).

24. Los casos son clasificados como: semidemocráticos y democráticos plenos (Mainwaring, Brinks y Pérez-Liñán, 2007).

25. Los errores estándar robustos son omitidos en la tabla para reducir espacio y que sea más sencilla la comparación de los resultados de los modelos. Quedan a disposición de quien lo solicite.

26. Por este motivo, se estimaron los modelos de la Tabla 5 como pooled. 


\section{REFERENCIAS BIBLIOGRÁFICAS}

ACEMOGLU, Daron y ROBINSON, James A. (2006), Economic origins of dictatorship and democracy. Cambridge: Cambridge University Press.

ANINAT, Cristóbal. (2006), "Balance de poderes legislativos en Chile.i Presidencialismo exagerado o base de un sistema político cooperativo?"; Política. Revista de Ciencia Política, vol. 47, pp. 128-148.

ARMESTO, Alejandra (2013), “Chile 1942-2010”. In: MAINWARING, Scott; PÉREZ-LIÑÁN, Aníbal. (2013), Democracies and Dictatorships in Latin America: Emergence, Survival, and Fall. Cambridge: Cambridge University Press. Informe anexo online. Disponible en: https://kellogg.nd.edu/sites/default/files/CountryReports.zip

BECK, Thorsten; CLARKE, George; GROFF, Alberto; KEEFER, Philip; WALSH, Patrick. (2012), "New tools in comparative political economy: The Database of Political Institutions". World Bank Economic Review, vol. 15, no. 1, pp. 165-176.

BOIX, Carles. (2003), Democracy and redistribution. Cambridge: Cambridge University Press.

BORMANN, Nils-Christian; GOLDER, Matt. (2013), “Democratic electoral systems around the world, 1946-2011". Electoral Studies, vol. 32, no. 2, pp. 360-369.

BUQUET, Daniel. (2007), "Entre la legitimidad y la eficacia: reformas en los sistemas de elección presidencial en América Latina". Revista Uruguaya de Ciencia Política, vol. 16, no. 1 , pp. $35-49$.

CASTAÑEDA-ANGARITA, Néstor. (2013), “Coding Costa Rica, 1944-2010”. In: MAINWARING, Scott; PÉREZ-LIÑÁN, Aníbal. (2013), Democracies and Dictatorships in Latin America: Emergence, Survival, and Fall. Cambridge: Cambridge University Press. Informe anexo online. Disponible en: https:/ / kellogg.nd.edu/sites/default/files/CountryReports.zip

CAREY, John y HIX, Simon. (2008), “Maximizing Representation and Accountability in the Design of Electoral Systems". Data Codebook Version.

CAREY, John y SHUGART, Matthew Soberg. (1998), Executive decree authority. Cambridge: Cambridge University Press.

COLOMER, Joseph. (2001), Instituciones politicas. Buenos Aires, Ariel.

COUTINHO, Marcelo. (2008), Crises institucionais e mudança política na América do Sul. Rio de Janeiro: Edição do autor.

DAHL, Robert (ed.). (1966), Political Oppositions in Western Democracies. New Haven: Yale University Press.

. (1973), Regimes and oppositions. New Haven: Yale University Press.

DOYLE, David; ELGIE, Robert (2016), "Maximizing the reliability of cross-national measures of presidential power". British Journal of Political Science, vol. 46, no 4, pp. 731-741.

DUVERGER, Maurice. (1957), Los partidos políticos. México DF: Fondo de Cultura Económica.

GALLAGHER, Michael. (1991), "Proportionality, disproportionality and electoral systems". Electoral Studies, vol. 10, no. 1, pp. 33-51. Disponible en: http:/ / www.tcd.ie/ Political_Science/Staff/Michael.Gallagher/ElSystems/index.php 


\section{Daniela Vairo}

GALLAGHER, Michael; MITCHELL, Paul. (eds.) (2008), The Politics of Electoral Systems. Oxford: Oxford University Press.

GAMBOA, Laura. (2017), "Opposition at the Margins: Strategies against the Erosion of Democracy in Colombia and Venezuela". Comparative Politics, vol. 49, no. 4, pp. 457-477.

GARCÍA, Craig; POLGA-HECIMOVICH, John. (2013), “Ecuador". In: MAINWARING, Scott; PÉREZ-LIÑÁN, Aníbal, Democracies and Dictatorships in Latin America: Emergence, Survival, and Fall. Cambridge: Cambridge University Press. Informe anexo online. Disponible en: https://kellogg.nd.edu/sites/default/files/CountryReports.zip

GARCÍA MONTERO, Mercedes. (2008), "Instituciones y actividad legislativa en América Latina”. Documentos CIDOB América Latina, vol. 23, no. 1, pp. 7-111.

HALL, Peter; TAYLOR, Rosemary. (1996), "Political science and the three new institutionalisms". Political Studies, vol. 44, no. 5, pp. 936-957.

HENISZ,Witold.(2002), “Theinstitutionalenvironmentfor infrastructureinvestment".Industrial and corporate change, vol. 11, no. 2, pp. 355-389.

. (2000), "The institutional environment for economic growth". Economics and politics, vol. 12, no. 1, pp. 1-31.

KARL, Terry. (1997), The paradox of plenty: Oil booms and petro-states. Berkeley: University of California Press.

LANZARO, Jorge. (ed.). (2012), "Presidencialismo con partidos y sin partidos. El presidencialismo en América Latina: debates teóricos y evolución histórica", in: Presidencialismo y parlamentarismo: América Latina y Europa Meridional: Argentina, Brasil, Chile, España, Italia, México, Portugal y Uruguay. Madrid, Centro de Estudios Políticos y Constitucionales.

LANZARO, Jorge. (2001), Tipos de presidencialismo y coaliciones politicas en América Latina. Buenos Aires, Clacso.

LIJPHART, Arend. (1999), Las democracias contemporáneas: un estudio comparativo. Madrid, Ariel.

LINZ, Juan José. (1987[1978]), El quiebre de los regímenes democráticos. Madrid, Alianza Editorial.

LIPSET, Seymour Martin. (1959), "Some social requisites of democracy: Economic development and political legitimacy". American Political Science Review, vol. 53, no. 1, pp. 69-105.

MAINWARING, Scott; PÉREZ-LIÑÁN, Aníbal. (2014), “La supervivencia de la democracia en América Latina (1945-2005)”. América Latina Hoy, vol. 68, pp. 139-168.

. (2013), Democracies and Dictatorships in Latin America: Emergence, Survival, and Fall. Cambridge: Cambridge University Press.

. (2009), ¿¿Por qué son importantes las regiones? Especificidades regionales y difusión de la democracia a escala regional". Revista SAAP, vol. 3, no. 3, pp. 523-566.

MAINWARING, Scott; BRINKS, Daniel; PÉREZ-LIÑÁN, Aníbal. (2007), “Classifying political regimes in Latin America, 1945-2004". In MUNCK, Gerardo (ed.), Regimes and Democracy in Latin America: Theories and Methods. Oxford: Oxford University Press. 
MAINWARING, Scott; SHUGART, Matthew. (2002), Presidencialismo y democracia en América Latina. Barcelona: Paidós.

MARCH, James G.; OLSEN, Johan P. (1983), “The new institutionalism: organizational factors in political life". American Political Science Review, vol. 78, no 3, pp. 734-749.

METCALF, Lee. (2000), "Measuring presidential power". Comparative Political Studies, vol. 33 , no. 5, pp. 660-685.

MORGENSTERN, Scott; POLGA-HECIMOVICH, John; SHAIR-ROSENFIELD, Sarah. (2013), “Tall, Grande, or Venti: Presidential Powers in the United States and Latin America". Journal of Politics in Latin America, vol., no. 2, pp. 37-70.

MUNCK, Gerardo. (2010), “Los orígenes y la durabilidad de la democracia en América Latina: Avances y retos de una agenda de investigación". Revista de Ciencia Política, vol. 30, no. 3, pp. 573-597.

O'DONNELL, Guillermo; SCHMITTER, Phillipe; WHITEHEAD, Lawrence. (1986). Transitions from authoritarian rule: Southern Europe. Baltimore: John Hopkins University Press.

PÉREZ-LIÑÁN, Aníbal; SCHMIDT, Nicolás; VAIRO, Daniela. (2019). Democratization. Forthcoming.

POWELL, G. Bingham. (2000), Elections as instruments of democracy: Majoritarian and proportional visions. New Haven: Yale University Press.

PRZEWORSKI, Adam. (2005), “Democracy as an Equilibrium”. Public Choice, vol. 123, no. 3-4, pp. 253-273.

. (1991), Democracy and the Market: Political and Economic Reforms in Eastern Europe and Latin America. New York: Cambridge University Press.

PRZEWORSKI, Adam; ALVAREZ, Michael; CHEIBUB, Jose Antonio; LIMONGI, Fernando. (1997), “What makes democracies endure?". Lua Nova: Revista de Cultura e Política, vol. 40-41, pp. 113-135.

ROSS, Michael L. (2001), “Does oil hinder democracy?”. World Politics, vol. 53, no.03, pp. 325-361.

SARTORI, Giovanni. (1966), Opposition and control problems and prospects. Government and Opposition, vol. 1, no. 2, pp. 149-154.

SHUGART, Matthew; CAREY, John. (1992), Presidents and Assemblies. Cambridge: Cambridge University Press.

TRAVERSA, Federico. (2016), "Por qué quiebran las democracias? Educación y conflicto de clase revisitados". Revista de Sociologia e Política, vol 24, no. 60, pp. 69-89.

TSEBELIS, George. (2006), Jugadores con veto: cómo funcionan las instituciones políticas. México: Fondo de Cultura Económica.

VAIRO, Daniela. (2017), “Oposición partidaria y (des)lealtad democrática en América Latina (1978-2010). POSTData, vol. 22, no. 1, pp. 273-304. 


\section{Daniela Vairo}

\section{RESUMO \\ Lealdade Democrática dos Partidos de Oposição na América Latina (1978-2010): Uma Explicação Institucionalista}

A pergunta central deste artigo é: sob que condições os partidos de oposição latino-americanos são leais ou desleais para com a democracia? Procurando dar resposta a esta questão, espera-se contribuir para a construção de uma teoria da lealdade democrática da oposição partidária para a América Latina pós-ditadura militar (1978-2010). Para isso, é proposta uma definição operacional da lealdade da oposição partidária e se apresenta a distribuição por país. Depois, é formulado um modelo teórico simples sobre sua variação, cuja hipótese central é que a lealdade democrática da oposição partidária depende em grande medida do grau de inclusão/exclusão institucional que se enfrenta. Afirma-se que os desenhos institucionais mais inclusivos são acompanhados por oposições partidárias leais, enquanto os sistemas mais excludentes derivam em oposições mais desleais. A exclusão institucional é operacionalizada em três dimensões: o sistema eleitoral legislativo, os poderes presidenciais e a dispersão do poder institucional. Finalmente, são testadas as hipóteses específicas por meio da estimação de modelos estatísticos. A análise estatística mostra que duas das três principais hipóteses têm um poder explicativo importante, portanto, pode-se afirmar que, institucionalmente, sistemas mais excludentes estão associados a oposições desleais. Isto tem importantes implicações em termos de desenho institucional e regime democrático.

Palavras-chave: oposição; instituições Políticas; democracia; partidos políticos; América Latina

\section{ABSTRACT \\ Democratic Loyalty of Opposition Parties in Latin America (1978-2010): An Institutionalist Explanation}

The main question of this article is: under what conditions are the Latin American opposition parties loyal or disloyal to democracy? Aiming to answer this question, we hope to contribute to the construction of a theory of the democratic loyalty of the opposition parties in Latin America after the military dictatorships (1978-2010). To achieve this goal, we propose an operational definition of the loyalty of the opposition parties, sorted out by countries. Next, we formulated a simple theoretical model of its variation, whose central hypothesis is that the democratic loyalty of the opposition parties depends to a large extent on the degree of institutional inclusion/exclusion it faces. It is argued that the more inclusive institutional designs are accompanied by loyal partisan oppositions, while the more exclusive systems 
result in more disloyal oppositions. Institutional exclusion is operationalized in three dimensions: the legislative electoral system, the presidential powers and the dispersion of institutional power. Finally, specific hypotheses are tested through the estimation of statistical models. Statistical analysis shows that two out of the three main hypotheses have important explanatory powers, so it can be said that, from the institutional point of view, systems that are more exclusive can be associated with unfair oppositions. This fact has important implications in terms of institutional design and democratic regime.

Keywords: opposition; political institutions; democracy; political parties; Latin America

\section{RÉSUMÉ \\ La Loyalté Démocratique des Partis d'Opposition à l'Amérique Latine (1978-2010): Une Explication Institutionnaliste.}

La question centrale de cet article est ceci: Dans quelles conditions les partis d'opposition latino-américains sont-ils loyaux ou déloyaux envers la démocratie? Pour répondre, on vise contribuer à la construction d'une théorie de la loyauté démocratique dans le cadre des oppositions partisans à l'Amérique Latine après les années des dictatures militaires (1978-2010). Pour cela, on utilise une définition opérationnelle de la loyauté de l'opposition partisan et on y propose de présenter sa répartition par pays. Ensuite, un modèle théorique simple de sa variation est formulée, dont l'hypothèse centrale est que la loyauté démocratique de l'opposition partisan dépend grossement du degré d'inclusion/exclusion institutionnelle à laquelle elle est confrontée. Il est avancé que les conceptions institutionnelles plus inclusives sont accompagnées d'oppositions partisanes loyales, tandis que les systèmes plus exclusifs dérivent d'oppositions plus déloyales. L'exclusion institutionnelle marche dans trois dimensions: le système électoral législatif, les pouvoirs présidentiels et la dispersion du pouvoir institutionnel. Au bout, les hypothèses spécifiques sont testées par l'estimation de modèles statistiques. L'analyse statistique montre que deux des trois hypothèses principales ont un pouvoir explicatif important. On peut donc dire que, de manière plus institutionnelle, des systèmes plus exclusifs sont associés à des oppositions injustes. À ce regard, il y a des implications importantes concernant à la conception institutionnelle et au régime démocratique.

Mots-clés: oposition; institutions politiques; démocratie; partis politiques; Amérique Latine 


\section{Daniela Vairo}

\section{RESUMEN:}

Lealtad Democrática de los Partidos de Oposición en América Latina (1978-2010): Una Explicación Institucionalista

La pregunta central de este artículo es: ¿sobre qué condiciones los partidos de oposición latinoamericanos son leales o desleales para la democracia? Buscando dar respuesta a esta cuestión, se espera contribuir a la construcción de una teoría de la lealtad democrática de la oposición partidaria para América Latina, pos dictadura militar (1978-2000). Para eso, es propuesta una definición operacional de la lealtad de la oposición partidaria y se presenta la distribución por país. Después, es formulado un modelo teórico simple sobre su variación, cuya hipótesis central es que la lealtad democrática de la oposición partidaria depende en gran medida del grado de inclusión/exclusión a la que se enfrenta. Se afirma que los diseños institucionales más inclusivos son acompañados por oposiciones partidarias leales, mientras que los sistemas más excluyentes derivan en oposiciones más desleales. La exclusión institucional es operacionalizada en tres dimensiones: el sistema electoral legislativo, los poderes presidenciales y la dispersión del poder institucional. Finalmente, se testean las hipótesis específicas por medio de la estimación de modelos estadísticos. El análisis estadístico muestra que dos de las tres hipótesis tienen un poder explicativo importante, sin embargo, se puede afirmar que, institucionalmente, sistemas más excluyentes están asociados a oposiciones desleales. Esto tiene importantes implicaciones en términos del diseño institucional y de régimen democrático.

Palabras clave: oposición; instituciones Políticas; democracia; partidos políticos; América Latina 